\title{
PERIODONTAL DISEASE-ASSOCIATED SNPS IN HEAD AND NECK CANCER IRRADIATION PATIENTS
}

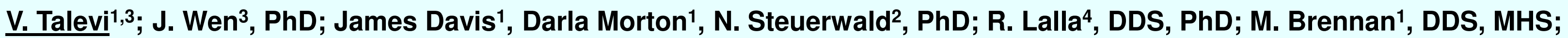 \\ X. Shi ${ }^{3}$, PhD; F. Mougeot ${ }^{1}$, PhD; J.L. Mougeot ${ }^{1,3}$, PhD
}

${ }^{1}$ Department of Oral Medicine, and ${ }^{2}$ Molecular Biology and Genomics Core Facility, Carolinas Medical Center, Atrium Health, Charlotte, NC, USA; ${ }^{3}$ College of Computing and Informatics, Department of Bioinformatics and Genomics, Charlotte, NC, USA;

${ }^{4}$ Section of Oral Medicine, University of Connecticut Health Center, Farmington, CT

\section{Abstract}

INTRODUCTION: Periodontal disease (PD) is a common oral complication in head and cancer patients (HNC) who undergo radiation therapy (RT) [1,2,3]. It has been previously shown that genetics play an important role in PD development.

OBJECTIVE: Our objective was to identify candidate single nucleotide polymorphisms (SNPs) associated with PD in radiation-treated HNC patients.

METHODS: Saliva DNA was extracted from HNC patients $(n=65)$ at baseline prior to RT. Clinical attachment loss (CAL) increment of $>0.2 \mathrm{~mm}$ was used as threshold to define PD development (PD group). A total of 44 patients among 65 developed PD. Exome sequencing and variant detection were performed using lon Proton sequencing platform. SNPs associated with PD were identified by logistic regression using PLINK v1.9 software (unadjusted p<0.05) [4]. Predominance of the homozygote SNP genotype for the alternate allele in patients who developed PD was determined using two-tailed z-test. STRING, PANTHER and GeneCodis programs were used for molecular network and gene ontology analyses.

RESULTS: A total of 454 candidate SNPs (385 genes) were identified, including 92 (60 genes) over-represented variant homozygous genotypes in PD group. Of these, 22 genes formed seven tight networks (90\% confidence level), including a collagen network and previously identified HLA-A and MMP2. These 22 genes were also integrated within a 66-gene network including 37 previously identified in PD association studies. Gene ontology analysis of this network showed the overrepresentation of biological processes such as collagen-dependent cell adhesion and the inflammatory response.

CONCLUSIONS: Polymorphisms affecting the integrity of the collagen matrix might confer susceptibility to PD in HNC patients undergoing RT.

\section{Results}

\section{Statistical results:}

1)

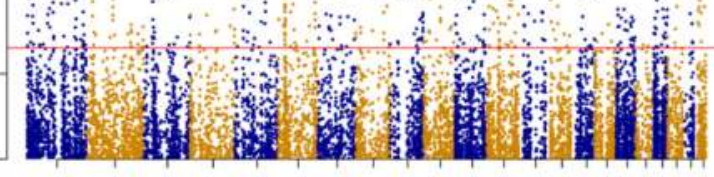

MANHATTAN PLOT: The red line denotes the significant level of $-\log (p)$ used in this study.

The SNPs above the red line denote 454 significant SNPs.

\section{2)}

Homozygosity test for the alternate allele (two-tailed z-test)

Reduced to 92 SNPS
$\Rightarrow 60$ genes
keeping genes less likely to
represent false positives

\section{Methods}

- Saliva DNA was extracted from HNC patients $(n=65)$ at baseline prior to $\mathrm{RT}=>$ Exome sequencing and variant detection were performed using lon Proton sequencing platform.

- CAL increment of $>0.2 \mathrm{~mm}$ was used as threshold to define to define PD development $=>$ A total of 44 patients among 65 developed PD.

- Logistic regression and two-tailed z-test were used to determine the SNPs associated with PD.

- Molecular network and gene ontology analyses were performed to determine biological significance of these SNPs.

\section{References}

1- Brennan MT, Treister NS, Sollecito TP, Schmidt BL, Patton LL, Mohammadi K, Long Simpson L, Voelker H, Hodges JS, Lalla RV. "Dental disease before radiotherapy in patients with head and neck cancer: Clinical Registry of Dental Outcomes in Head and Neck Cancer Patients." J Am Dent Assoc., 148(12):868-877, 2017

2- Lalla RV, Treister N, Sollecito T, Schmidt B, Patton LL, Mohammadi K, Hodges JS, Brennan MT; OraRad Study Group. "Oral complications at 6 months after radiation therapy for head and neck cancer." Oral Dis., 23(8):1134-1143, 2017 3- Machtei EE, Norderyd J, Koch G, Dunford R, Grossi S, Genco RJ. The rate of periodontal attachment loss in subjects with established

4- PLINK website: http://zzz.bwh.harvard.edu/plink/contact.shtm enes had SNPs homozygous for the alternate allele and were over-represented in HNC patients who developed PD $=>$ potentially conferring SUSCEPTIBILITY (pink circle)

Collagen network (red D development HLA-A (VeUl[OW) and genes have been previously identified as potential biomarkers for PD.

Gene ontology analysis entified the biological processes inflammatory response (orange dashed line).

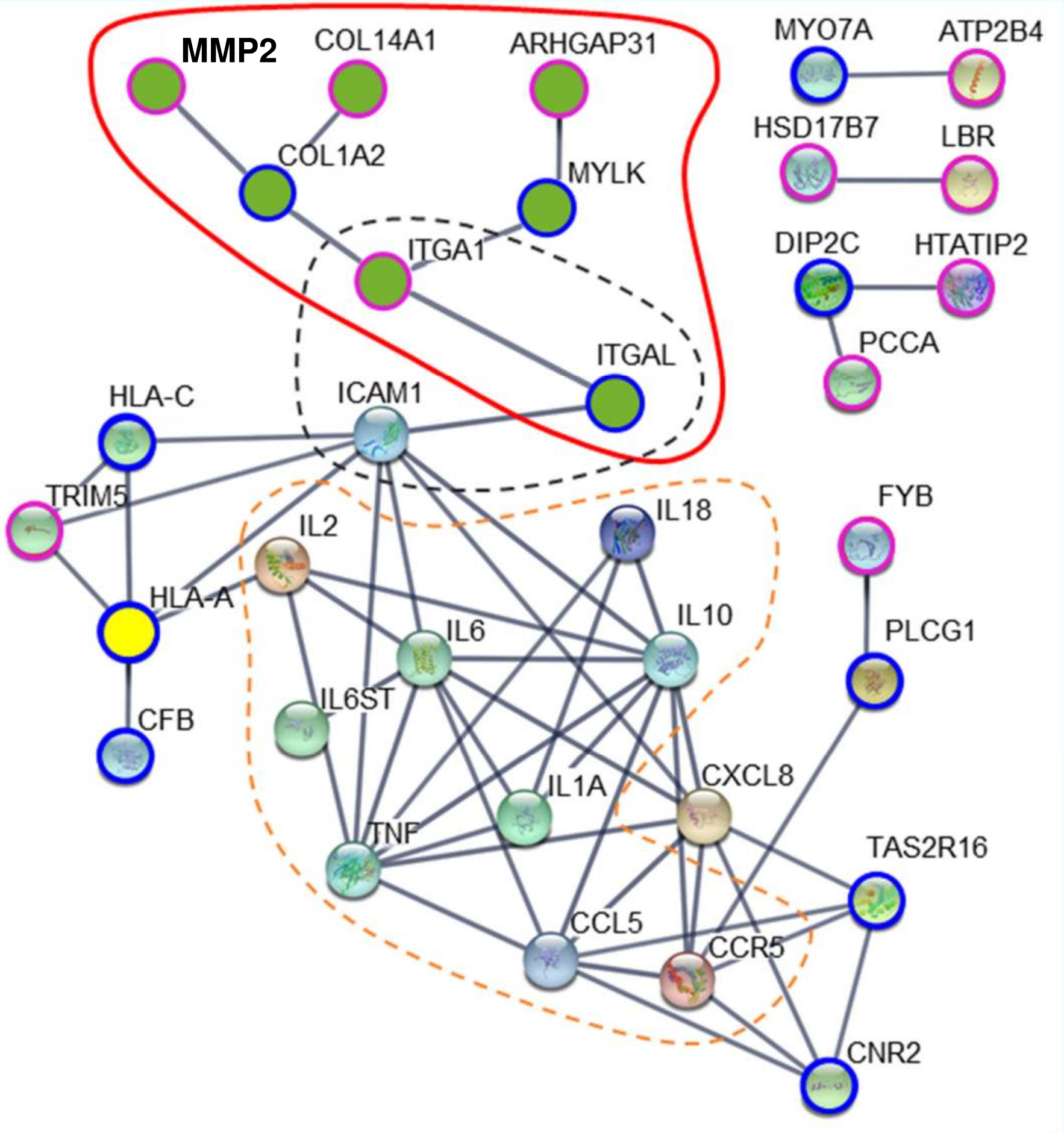

- Conclusion patients were identified.

- Polymorphisms located in collagen pathway genes might confer susceptibility to PD of HNC patients undergoing RT.

- The study could potentially contribute to the development of risk assessment strategies and personalized treatment in the HNC patient population.

\section{Acknowledgments}

